ORIGINAL ARTICLE

\title{
Relationship between probable nosocomial bacteraemia and organisational and structural factors in UK neonatal intensive care units
}

\author{
The UK Neonatal Staffing Study Group*
}

Qual Saf Health Care 2005;14:264-269. doi: 10.1136/qshc.2004.012690

See end of article for authors' affiliations

Correspondence to: Dr G J Parry, Health Services Research, School of Health and Related Research, University of Sheffield, Sheffield S1 4DA, UK; g.parry@ sheffield.ac.uk

Accepted for publication 16 May 2005
Objectives: To assess the relationship between organisational and structural factors of UK neonatal intensive care units (NICUs) with risk adjusted probable nosocomial bacteraemia.

Design of study: A prospective observational study of infants concurrently admitted to 54 randomly selected UK NICUs between March 1998 and April 1999.

Results: Of the 13334 infants admitted, 402 (2.97\%) had probable nosocomial bacteraemia. The median unit level percentage of infants with probable nosocomial bacteraemia was $2.48 \%$ (minimum $0 \%$, maximum 9\%). The risk adjusted odds of probable nosocomial bacteraemia were increased by $1.13(95 \%$ $\mathrm{Cl} 1.07$ to 1.20 ) for each additional level 1 cot per hand washbasin and decreased by 0.53 (95\% $\mathrm{Cl} 0.35$ to 0.79 ) in infants admitted to units with an NICU infection control nurse compared with units without. There was no relation with an increase in the floor space of the unit per cot lodds ratio $0.99195 \% \mathrm{Cl} 0.98$ to 1.00 ) per $\mathrm{m}^{2}$ ) or with the quality of hand washing signs (odds ratio 1.04 (95\% Cl 0.93 to 1.16$)$ per increase in quality score).

Conclusions: There is widespread variation in rates of probable nosocomial bacteraemia in UK NICUs. Probable nosocomial bacteraemia is reduced in units with a dedicated infection control nurse and with the presence of more hand washbasins. Further research is required to identify methods to eliminate nosocomial bacteraemia.
$\mathrm{T}$

he Harvard Medical Practice Study found that hospital acquired infection is the second leading cause of adverse events. ${ }^{1}$ In neonatal intensive care, hospital acquired infection can lead to increased illness severity, length of stay, neurodevelopmental and growth impairment, and mortality and thus incur major additional healthcare costs. ${ }^{2-4}$

Previous evidence indicates that the incidence of hospital acquired infection, measured as nosocomial bacteraemia in neonatal intensive care units (NICUs), varies considerably between units. In a study of six NICUs in the United States, $19.1 \%$ of infants of less than $1500 \mathrm{~g}$ were found to have a nosocomial bloodstream infection (range 8.5-42\%). ${ }^{5}$ Another study found that $65 \%$ of infants of $400-1000 \mathrm{~g}$ admitted to academic medical centres in the United States developed at least one infection during their hospital stay. ${ }^{3}$

There is evidence that variations in the rates of hospital acquired infections are linked to the hand hygiene of staff. ${ }^{6}$ Standards of hand hygiene are related to multiple factors including staff behaviour and the structural environment within which they work..$^{7-9}$ For example, the presence of sufficient and accessible hand washing facilities, the presence of physical signs to guide staff on hand hygiene, the amount of space between cots, and the presence of a healthcare professional whose role it is to monitor infection rates and implement hospital acquired infection reduction initiatives may all impact on the standards of hand hygiene and consequently on the rate of nosocomial bacteraemia within an NICU.

As part of the UK Neonatal Staffing Study (UKNSS, see box 1$),{ }^{10}$ we aimed to examine prospectively the relationship between probable nosocomial bacteraemia in all infants admitted and specific unit organisational and structural

* Members of the UK Neonatal Staffing Study Group are given at the end of the paper. characteristics that may impact on the hand hygiene of the unit: the average floor space per intensive care cot, average number of level 1 cots per hand washbasin, quality measures of hand washing signs, and whether there is an infection control nurse or link nurse whose role it is to monitor infection rates and implement infection control initiatives for the unit.

Box 1 The United Kingdom Neonatal Staffing Study (UKNSS)

The United Kingdom Neonatal Staffing Study (UKNSS) prospectively evaluated whether patient volume, staffing levels, and workload were related to risk adjusted outcome.

All 186 UK neonatal intensive care units (NICUs) were stratified into 12 types in a $3 \times 2 \times 2$ factorial matrix according to volume of patients, nursing provision, and neonatal consultant provision. Three primary outcomes were prospectively evaluated in 13515 infants of all birth weights consecutively admitted between March 1998 and April 1999 to 54 NICUs randomly selected from this factorial matrix: (1) hospital mortality, (2) mortality or cerebral damage, and (3) nosocomial bacteraemia. Risk adjusted mortality and mortality or cerebral damage were unrelated to patient volume or staffing provision, but probable nosocomial bacteraemia was less frequent in NICUs with low neonatal consultant provision (odds ratio $0.65,95 \% \mathrm{Cl} 0.43$ to 0.98 ).

The UKNSS also aimed to examine prospectively the relationship between hospital acquired infection (measured as probable nosocomial bacteraemia in all infants admitted) and specific organisational characteristics that may impact on the hand hygiene of the unit. 


\section{METHODS}

\section{Unit recruitment}

The UKNSS recruited a representative sample of 54 NICUs according to a $3 \times 2 \times 2$ factorial design (box 1). Census data collected in 1997 identified 186 UK hospitals with NICUs and allowed unit stratification of these units into 12 types using a 3 (high, medium and low volume) $\times 2$ (high and low neonatal consultant provision) $\times 2$ (high and low nursing provision) factorial design. ${ }^{10}$ Fifty four NICUs randomly selected from each type were invited to participate in the prospective phase.

\section{Infant recruitment}

All infants aged less than 1 month corrected for gestation consecutively admitted between March 1998 and April 1999 to the 54 NICUs were eligible for inclusion. Clinical data were abstracted from clinical records for all infants during the observation period.

\section{Outcome}

The primary outcome in the current study was probable nosocomial bacteraemia defined as those infants who, following negative blood cultures from birth up to 48 hours, had at least one positive blood culture during the period from 48 hours after birth to discharge home. Infants were recorded as having either one or more episodes of probable nosocomial bacteraemia or none at all. For transferred infants, probable nosocomial bacteraemias were attributed to the hospital of care when hospital of birth transferred $\leqslant 24$ hours after birth. Transfers $>24$ hours were not attributable to the receiving hospital in analysis by hospital of care.

\section{Organisational and structural characteristics}

Before the start of infant recruitment, participating NICUs were visited by two members of the Writing Committee (WOTM and JT). At these visits, data on the floor space, number of hand washbasins (within the immediate level 1 cot care area), and number and level/type of cots was recorded. The average floor space and number of hand washbasins per intensive care cot were calculated by dividing floor space and number of hand washbasins, respectively, by the number of cots. Unit directors provided information on whether the unit had an appointed infection control nurse or link infection control nurse. The infection control nurse was someone in the NICU establishment with that identified role and a specific remit to facilitate improvement of infection control practice within the unit. A link (infection control) nurse was a nurse with that remit for the NICU, but who also undertook the role of monitoring and promoting infection control more widely-for example, in a number of clinical settings throughout the hospital. The measure for quality of hand washing signs was to note the number of hand washing signs and their specific content at site visit. The score was derived by adding scores for each sign, where a sign scored 1 for clear instructions on how to wash hands effectively and 1 for instruction of when to wash hands.

Table 1 Organisms isolated during the study period

\begin{tabular}{ll}
\hline Organism & $\begin{array}{l}\text { Total number of episodes of } \\
\text { probable nosocomial } \\
\text { bacteraemia }\end{array}$ \\
\hline Coagulase negative staphylococcus & 306 \\
Group B streptococcus & 23 \\
Other Gram positive & 64 \\
Gram negative & 35 \\
Fungal & 13 \\
\hline
\end{tabular}

\section{Data analysis}

For descriptive purposes, the number of infants admitted between occurrences of probable nosocomial bacteraemia was calculated for each unit, indicating how frequently probable nosocomial bacteraemia occurred. ${ }^{11}$ For each infant who developed probable nosocomial bacteraemia, the number of admissions between their date of admission and the date of admission of the next infant admitted who developed probable nosocomial bacteraemia was recorded as a proxy measure to indicate the frequency of occurrence of probable nosocomial bacteraemia. The frequency was compared using this measure between units grouped by unit hand hygiene variables using the median (and quartiles) and MannWhitney test.

Using logistic regression techniques, after adjusting for illness severity, probable nosocomial bacteraemia was tested for independent association with average floor space per intensive care cot, average number of hand washbasins per intensive care cot, appointment of an infection control nurse or link nurse for the unit, and the quality measures of hand washing signs. Generalised estimating equations were used to correct the standard errors of hospital level variables for the effect of within hospital correlation. ${ }^{12}{ }^{13}$ The illness severity of each infant was measured using variables identified in a previously described model (gestational age, size for gestation, sex, mode of delivery, diagnosis category, and maternal treatment with antenatal steroids). ${ }^{10}$

Confounding due to the frequency and timing of blood cultures by each unit was assessed in two ways. Firstly, the correlation between the ratio of positive blood cultures to all blood cultures after 48 hours and the rate of probable nosocomial bacteraemia after 48 hours was examined. Secondly, the correlation between the ratio of positive blood cultures to all blood cultures after 48 hours and each of the hand hygiene factors was examined.

\section{RESULTS}

Of 13334 eligible infants admitted to 54 NICUs between March 1998 and April 1999, the overall median (quartiles) birth weight was $2500 \mathrm{~g}(1855,3280)$, ranging from $1950 \mathrm{~g}$ $(1310,3010)$ to $2860 \mathrm{~g}(2090,3475)$. The overall median (quartiles) gestational age was $36(33,39)$ completed weeks, ranging from $33(31,37)$ to $38(35,40)$ completed weeks.

A total of 441 episodes of probable nosocomial bacteraemia were identified in 402 infants (2.97\% of infants observed). Of the organisms isolated (table 1), coagulase negative staphylococcus accounted for 306 (76.1\% of infants with probable nosocomial bacteraemia). In 1433 infants of less than 31 weeks gestation, 274 episodes of probable nosocomial bacteraemia were identified in 259 (18.1\%) infants. Figure 1

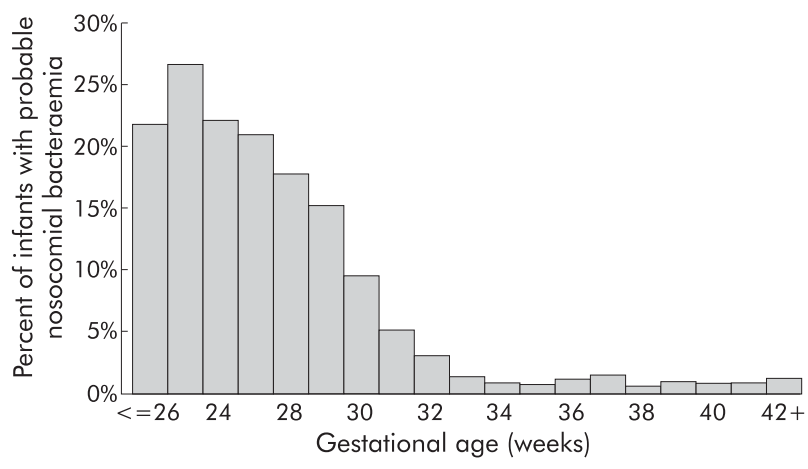

Figure 1 Percentage of infants with probable nosocomial bacteraemia by gestational age (completed weeks gestation at birth). 

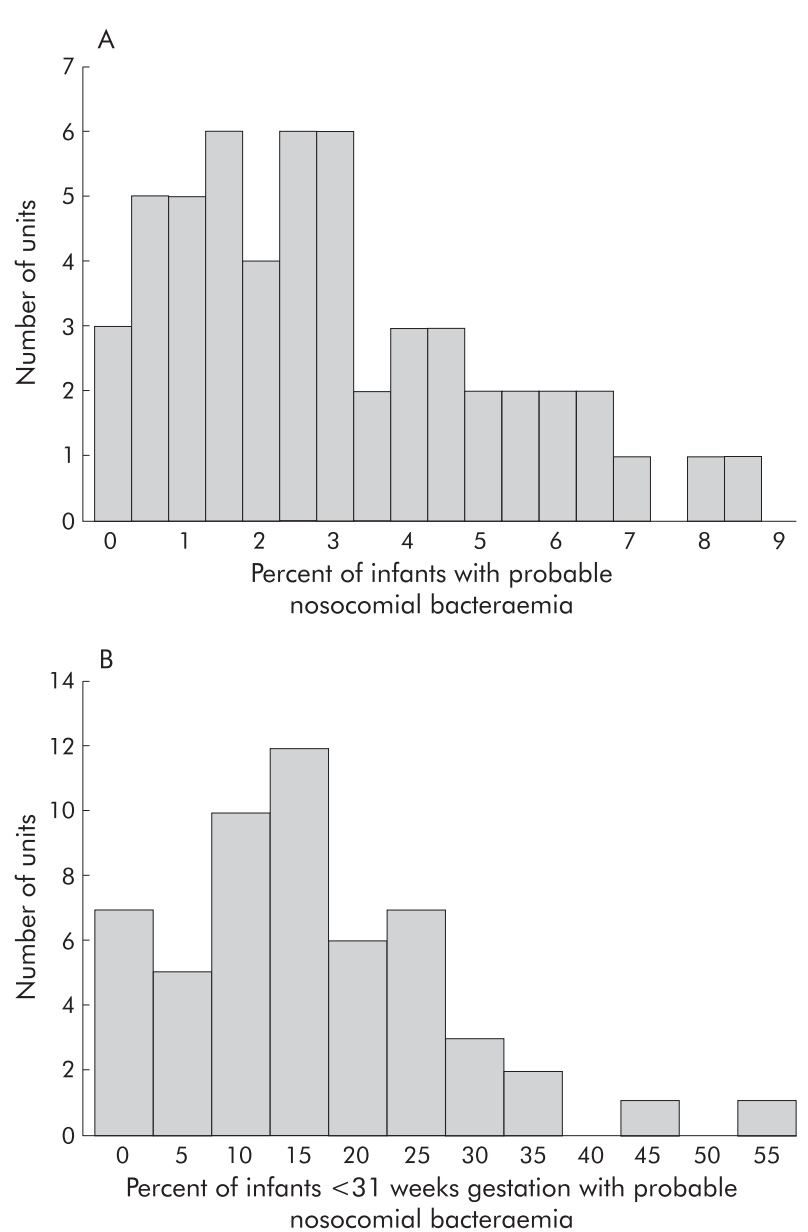

Figure 2 (A) Percentage of infants with probable nosocomial bacteraemia by neonatal intensive care unit. (B) Percentage of infants of less than 31 completed weeks gestation with probable nosocomial bacteraemia by neonatal intensive care unit.

shows the percentage of infants who developed probable nosocomial bacteraemia by gestational age.

Variation in probable nosocomial bacteraemia by unit Among the 54 units, the median proportion of infants with probable nosocomial bacteraemia was $2.48 \%$ (quartiles $1.27 \%, 4.3 \%)$, varying from $0 \%$ in three units to $9 \%$ in one unit. The distribution of probable nosocomial bacteraemia by NICU is shown in fig 2A. Over all units, the median number of admissions between cases of probable nosocomial bacteraemia was 14 (quartiles 6, 29). This varied from a gap of 8 admissions (quartiles 5,16 ) in one unit to 64 (quartiles 15 , 112 ) in another unit. The median proportion of infants with coagulase negative staphylococcus in all the units was $1.53 \%$ (quartiles $0.74 \%, 3.16 \%$ ).
To allow comparison with studies focusing on very premature infants, the distribution of probable nosocomial bacteraemia by NICU for infants of less than 31 completed weeks gestation is shown in fig $2 \mathrm{~B}$. Here the median proportion of infants with probable nosocomial bacteraemia was $13.3 \%$ (quartiles $8.7 \%, 22.7 \%$ ), varying from $0 \%$ in seven units to $54.2 \%$ in one unit.

\section{Average floor space per intensive care cot}

Data on complete floor space per intensive care cot were available for 48 units. The median (quartiles) floor space per level 1 cot was $10.96 \mathrm{~m}^{2}(7.30,16.13)$. Table 2 shows that the risk adjusted odds of probable nosocomial bacteraemia were not related to the average floor space per level 1 cot (odds ratio 0.99 (95\% CI 0.98 to 1.00 ) for each $\mathrm{m}^{2}$ ).

\section{Average number of hand washbasins per intensive care cot}

The total number of level 1 cots was divided by the number of hand washbasins within the level 1 care area for each NICU. The median (quartiles) number of level 1 cots per hand washbasin was $2.38(2,4)$, with an associated percentage of infants with probable nosocomial bacteraemia of $3.15 \%$ $(2.46 \%, 3.84 \%)$. Nine units had one or fewer level 1 cots per hand washbasin. Table 2 shows that the adjusted odds of probable nosocomial bacteraemia were increased by 1.13 (95\% CI 1.07 to 1.20 ) for each additional level 1 cot per hand washbasin (fig 3). For units with more than one level 1 cot per hand washbasin, the median number of admissions between cases of probable nosocomial bacteraemia was 13 (5, $25)$ compared with 20 admissions $(9,38)$ in units with one or fewer level 1 cots per hand washbasin $(p=0.005)$.

\section{Appointment of an infection control nurse or link nurse for the unit}

Of the 54 units, $42(78 \%)$ had an appointed NICU infection control nurse or link nurse. In units with an infection control

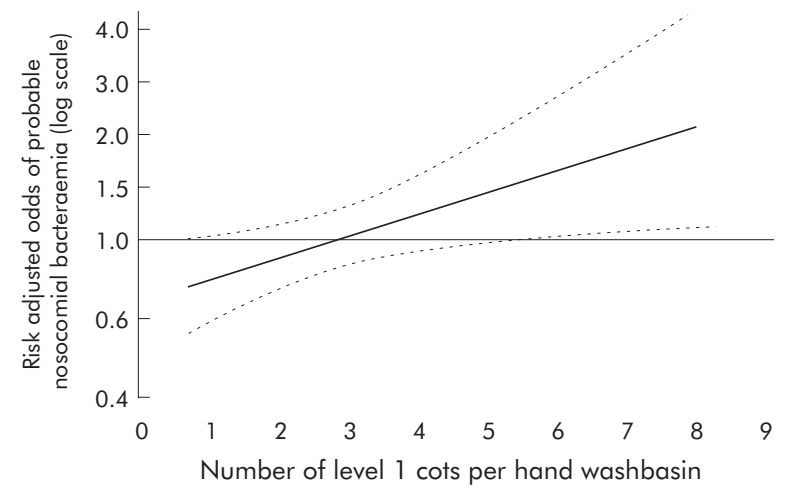

Figure 3 Percentage of infants with probable nosocomial bacteraemia by number of level 1 cots per hand washbasin in the unit of care.

Table 2 Odds ratios for probable nosocomial bacteraemia by each organisational and structural unit characteristic

\begin{tabular}{llll}
\hline & Odds ratio & $95 \% \mathrm{Cl}$ & p value \\
\hline Illness severity (birth model) & 0.60 & $(0.20$ to 0.62$)$ & $<0.001$ \\
Unit has infection control or link nurse & 0.53 & $(0.35$ to 0.79$)$ & 0.030 \\
No of level l cots per hand washbasin & 1.13 & $(1.07$ to 1.20$)$ & 0.006 \\
Floor space per level 1 cot $\left(\mathrm{m}^{2}\right)$ & 0.99 & $(0.98$ to 1.00$)$ & 0.234 \\
Quality score for hand washing & 1.04 & $(0.93$ to 1.16$)$ & 0.537 \\
\hline
\end{tabular}


nurse, 335 of 11264 infants (2.97\%) had probable nosocomial bacteraemia. In units with an appointed infection control or link nurse the median percentage of infants who had probable nosocomial bacteraemia was $2.41 \%$, ranging from $0 \%$ in two units $(0 / 100$ and $0 / 120$, respectively) to $7.87 \%$ $(28 / 328)$ in one unit. In units without an infection control nurse, 131 of 3079 infants $(4.25 \%)$ had probable nosocomial bacteraemia. In units without an appointed infection control or link nurse the median percentage of infants who had probable nosocomial bacteraemia was $3.91 \%$, ranging from $0 \%$ in one unit $(0 / 59)$ to $8.65 \%(23 / 266)$ in one unit. Alternatively, for units without an infection control nurse, the median number of admissions between cases of probable nosocomial bacteraemia was 10 (quartiles 4, 21) compared with 15 (quartiles 7,33 ) in units with an infection control nurse $(\mathrm{p}=0.003)$.

Table 2 shows that the risk adjusted odds of probable nosocomial bacteraemia were reduced by 0.53 (95\% CI 0.35 to 0.79 ) in units with an appointed infection control nurse or link nurse.

\section{Quality measures of hand washing signs}

The median (quartiles) quality measures of hand washing signs was $1.5(0,3)$ ranging from 0 in 20 units to 12 in one unit. Table 2 shows that the risk adjusted odds of probable nosocomial bacteraemia were not related to the quality measures of hand washing signs ( 1.04 (95\% CI 0.93 to 1.16) per increase of 1 in the quality score).

\section{Assessment of confounding due to the frequency and timing of blood cultures}

The ratio of positive blood cultures to all blood cultures after 48 hours for each unit was not found to be statistically associated with the rate of probable nosocomial bacteraemia $(p=0.181)$, average floor space per intensive care cot $(p=0.699)$, average number of level 1 cots per hand washbasin $(p=0.127)$, appointment of an infection control nurse or link nurse for the unit $(p=0.267)$, and quality measures of hand washing signs $(p=0.154)$, which suggests that there was no major confounding in the identification of probable nosocomial bacteraemia associated with the frequency of blood culture testing in each unit.

\section{DISCUSSION}

This prospective study has shown wide variation in rates of probable nosocomial bacteraemia in infants of all gestational ages in NICUs in the UK. The levels of probable nosocomial bacteraemia were similar to those in other developed countries. $^{14-16}$ The risk of probable nosocomial bacteraemia was reduced in units with a dedicated infection control nurse and with the presence of more hand washbasins. No association was detected between the floor area of the units or quality measures of current hand washing signs and rates of probable nosocomial bacteraemia.

This is a prospective observational study and we cannot rule out possible bias in the data from an uncontrolled study design. We also cannot adjust for the impact of increased rates of contaminated blood cultures in some NICUs. In addition, the study only provides information on what structures or organisational features may be associated with probable nosocomial bacteraemia and cannot determine what activities performed by an infection control nurse lead to reductions in nosocomial bacteraemia. For this, detailed ethnographic studies or larger randomised controlled trials are required. The reliability of the quality measures of hand washing signs is questionable because two NICUs stated they had a policy to minimise all wall signs but regularly used staff training for hand washing protocols. Furthermore, it is difficult to interpret cause and effect in relation to the quality of measures of hand washing-for example, units with a high perceived level of nosocomial bacteraemia may put up additional signs in response to previous occurrences of infections.

The UKNSS showed that there were higher rates of probable nosocomial bacteraemia in NICUs with a higher consultant provision and designated consultant for the clinical management of units, but no significant relationship with nursing provision. ${ }^{12}$ Other studies have suggested that invasive procedures, overcrowding, and understaffing in periods of increased workload, poorer compliance with hand washing by clinicians compared with nursing staff, and poorer compliance within intensive care settings may provide an explanation for this finding. ${ }^{6}{ }^{17}{ }^{18}$ It is likely that, in a busy and overcrowded clinical setting, having a dedicated clinical member of staff whose specific role is to improve infection control procedures within their setting will help decrease nosocomial bacteraemia. ${ }^{19}$

As a result of recent studies, current recommendations in the UK and the US encourage hand hygiene using basin-free alcohol rub to supplement the use of soap and water.9-11 2021 None of the recommendations specify the number or positioning of the alcohol rub. Our findings suggest that having a hand washbasin available at the cot side of every level 1 cot may reduce probable nosocomial bacteraemia. Although our study did not specifically examine the availability of alcohol rub in the units, it may be that having alcohol rub available to clinical staff at the side of every level l cot will lead to a further reduction in nosocomial bacteraemia. Other studies have demonstrated the use of hospital wide programmes aimed at changing staff behaviour in order to reduce levels of hospital acquired infection and these may also be applicable to the NICU. ${ }^{72}$ In addition, collaborative quality improvement methods have identified and recommended multifactorial methods of improving clinical practice that may reduce rates of nosocomial bacteraemia in NICUs. ${ }^{89}$

The current results are based on a large study of 54 representative UK NICUs. In other healthcare settings such large scale data collection, allowing an examination of relationships between hospital structures and nosocomial bacteraemia, has not been undertaken so less robust evidence is available to guide decision makers. This highlights the benefits to clinicians, managers, and policymakers that can be gained from undertaking standardised data collection across large numbers of units.

\section{Key messages}

- Rates of hospital acquired infection measured as probable nosocomial bacteraemia vary widely in UK neonatal intensive care units.

- The risk of an infant acquiring probable nosocomial bacteraemia appears to decrease with the presence of an infection control or link nurse and with more hand washbasins in the unit.

- Consideration should be given to ensuring every clinical area has a healthcare professional whose sole and specific remit is to decrease rates of hospital acquired infection and that hand washbasins and alcohol rub are readily available at every cot or bedside.

- Further research is required to identify methods that aim to eliminate nosocomial bacteraemia. 
The results of the current study may inform a wider range of healthcare settings and other forms of infection, particularly for vulnerable patients. The widespread differences in rates of probable nosocomial bacteraemia in NICUs are a major cause for concern. In the light of our results and other literature, to reduce the variation in, and overall rate of, nosocomial bacteraemia, we recommend that consideration should be given to supporting behavioural based educational and quality improvement initiatives by meeting the following structural based requirements:

- Every clinical area should identify a healthcare professional whose sole and specific remit is to decrease rates of nosocomial infection.

- Hand washbasins and alcohol rub should be readily available at every cot or bedside.

- Infection rates and efforts to reduce them should be fed back to all staff rapidly and reliably. ${ }^{23}$

This study provides some evidence for ways in which rates of probable nosocomial bacteraemia may be reduced, but it does not prescribe how they may be reduced to zero. ${ }^{24}$ For this, a complete systems based re-think is required. Facilitating such a system will require greater empowerment of nursing and junior clinical staff to speak up if they see another member of the clinical staff not following hand hygiene protocols, and may also require that patients-or, in this case, the parents or guardians of patients-are empowered to speak up too..$^{25-28}$

\section{ACKNOWLEDGEMENTS}

The authors thank all participating neonatal units and staff for their support, and link audit nurses, nurse managers and clinicians who collected prospective data on our behalf. They also thank Don Goldmaan for his advice. The views expressed in this paper are those of the Writing Committee.

This study was funded by the NHS R\&D Executive, Mother and Child Health Programme (grant number MCH:6-7) and endorsed by the British Association of Perinatal Medicine, the Neonatal Nurses Association (UK), and Scottish Neonatal Nurses Group. Gareth Parry was supported in writing this paper by The Commonwealth Fund, a national private foundation based in New York City that supports independent research on health and social issues. The views presented here are those of the author and not necessarily those of The Commonwealth Fund, its director, officers, or staff.

The authors have no conflict of interest to declare.

Gareth Parry planned and coordinated the writing of the paper, undertook the analysis, contributed to the study design and contributed to all sections of the paper. Janet Tucker contributed to the study design and contributed to all sections of the paper. William Tarnow-Mordi contributed to the study design and contributed to all sections of the paper.

UK Neonatal Staffing Study Writing Committee: G J Parry, Health Services Research, ScHARR, University of Sheffield; J S Tucker, Dugald Baird Centre for Research on Women's Health, Department of Obstetrics and Gynaecology, University of Aberdeen; W O Tarnow-Mordi, University of Sydney, Sydney, Australia

UK Neonatal Staffing Study Steering Group: N Marlow (Chair), Department of Child Health, University of Nottingham; H Baumer, Department of Child Health, Derriford Hospital, Plymouth; M Boen, SCBU, Derby City Hospital; K Hamilton, Dumfries and Galloway Health Board; J Meran, Neonatal Unit, Good Hope Hospital, Stourbridge; D Milligan, Neonatal Unit, Royal Victoria Infirmary, Newcastle; C McCabe, ScHARR, University of Sheffield; J Nicholl, Medical Care Research Unit, University of Sheffield; P Nicolson, ScHARR, University of Sheffield; M Redshaw, Institute of Child Health, Royal Hospital for Sick Children, Bristol; K Rowan, ICNARC, Tavistock Square, London; J Scott, Neonatal Unit, Queen Mother's Hospital, Glasgow; J Smith, SCBU, Princess Margaret Hospital, Swindon

Participating NICUs: Airedale General Hospital, Antrim Hospital, Arrowe Park Hospital, Birch Hill Hospital, Burnley General Hospital, City Hospital Birmingham, Derby City Hospital NHS Trust, Dewsbury
District Hospital, Erne Hospital, Fairfield General Hospital, Farnborough Hospital, Gloucestershire Royal Hospital, Good Hope Hospital, Greenwich Healthcare Trust, Grimsby Maternity Hospital, Hereford County Hospital, Huddersfield Royal Infirmary, Jersey Maternity Hospital, Kent \& Canterbury Hospital, Kettering General Hospital, King's Mill Hospital, Norfolk \& Norwich Hospital, North Devon District Hospital, Northampton General Hospital, Northern General Hospital Sheffield, Northwick Park Hospital, Nottingham University Hospital, Peterborough District Hospital, Prince Charles Hospital, Queen Elizabeth Hospital Gateshead, Rotherham District General Hospital, Royal Alexandra Hospital, Royal Devon \& Exeter Hospital, Royal Hampshire County Hospital, Royal Oldham Hospital, Royal Preston Hospital, Royal Surrey County Hospital, Royal United Hospital, Salisbury NHS Trust, Scunthorpe General Hospital, South Cleveland Hospital, South End Hospital, Southern General Hospital, St George's Hospital, St Mary's Hospital, St Paul's Hospital, St Peter's Hospital, St Thomas' Hospital, Stoke Mandeville Hospital, The Ipswich Hospital NHS Trust, William Harvey Hospital, Wordsley Hospital, Wycombe General Hospital, York District Hospital.

\section{REFERENCES}

1 Leape LL, Brennan TA, Laird N, et al. The nature of adverse events in hospitalized patients: results of the Harvard Medical Practice Study II. N Engl J Med 1991;324:377-84.

2 Fowlie PW, Gould CR, Parry GJ, et al. CRIB (clinical risk index for babies) in relation to nosocomial bacteraemia in very low birth weight or preterm infants. Arch Dis Child 1996:75:F49-52.

3 Stoll BJ, Hansen NI, Adams-Chapman I, et al. Neurodevelopmental and growth impairment among extremely low-birth-weight infants with neonatal infection. JAMA, 2004 17, 292:2357-65.

4 Stoll BJ, Hansen N. Infections in VLBW infants: studies from the NICHD Neonatal Research Network. Semin Perinatol 2003;27:293-301.

5 Brodie SB, Sands KE, Gray JE, et al. Occurrence of nosocomial bloodstream infections in six neonatal intensive care units. Pediatr Infect Dis $J$ 2000; 19:56-65.

6 Pittet D, Mourouga P, Perneger TV. Compliance with hand washing in a teaching hospital. Ann Intern Med 1999:130:126-30.

7 Pittet D, Hugonnet S, Harbarth S, et al. Effectiveness of a hospital-wide programme to improve compliance with hand hygiene. Lancet 2000;356:1307-12

8 Kilbride HW, Powers R, Wirtschafter DD, et al. Evaluation and development of potentially better practices to prevent neonatal nosocomial bacteremia. Pediatrics 2003;111:e504-18.

9 Horbar JD, Rogowski J, Plsek PE, et al. Collaborative quality improvement for neonatal intensive care. NIC/Q Project Investigators of the Vermont Oxford Network. Pediatrics 2001;107:14-22.

10 UK Neonatal Staffing Study Group. A prospective evaluation of patient volume, staffing and workload in relation to risk-adjusted outcomes in a random, stratified sample of all UK neonatal intensive care units. Lancet 2002;359:99-107.

11 Benneyan JC, Lloyd RC, Plsek PE. Statistical process control as a tool for research and healthcare improvement. Qual Saf Health Care 2003;12:458-64.

12 Ukoumunne OC, Gulliford MC, Chinn S, et al. Methods for evaluating areawide and organisation-based interventions in health and health care: a systematic review. Health Technol Assessment 1999:3(5).

13 StataCorp. Statistical Software: Release 8.0. College Station, TX: Stata Corporation, 2003

14 Lopez Sastre JB, Coto Cotallo D, Fernandez Colomer B. Neonatal sepsis of nosocomial origin: an epidemiological study from the "Grupo de Hospitales Castrillo". J Perinatal Med 2002;30:149-57.

15 Stoll BJ, Hansen N, Fanaroff AA, et al. Late-onset sepsis in very low birth weight neonates: the experience of the NICHD Neonatal Research Network. Pediatrics 2002;110:285-91.

16 Ferguson JK. Gill A. Risk-stratified nosocomial infection surveillance in a neonatal intensive care unit: report on 24 months of surveillance, J Paediatr Child Health 1996;32:525-31.

17 Brodie SB, Sands KE, Gray JE, et al. Occurrence of nosocomial bloodstream infections in six neonatal intensive care units. Pediatr Infect Dis $J$ 2000;19:56-65.

18 Harbarth S, Sudre P, Dharan S, et al. Outbreak of Enterobacter cloacae related to understaffing, overcrowding and poor hygiene practices. Inf Control Hosp Epidemiol 1999;20:598-603.

19 Teare EL, Peacock AJ, Dakin H, et al. Build your own infection control link nurse: an innovative study day. J Hosp Infect 2001;48:312-9.

20 Hugonnet S, Perneger T, Pittet D. Alcohol-based handrub improves compliance with hand hygiene in intensive care units. Arch Intern Med 2002; 162:1037-43.

21 Pratt RJ, Pellowe C, Loveday HP, and the Epic Guideline Development Team, et al. The Epic project: developing national evidence-based guidelines for preventing healthcare associated infections Phase 1: Guidelines for preventing hospital-acquired infections. J Hosp Infect $2001 ; 47($ Suppl):S1-82.

22 Kurlat I, Corral G, Oliveira F, et al. Infection control strategies in a neonatal intensive care unit in Argentina. J Hosp Infect 1998;40:149-54.

23 Burke JP. Infection control - a problem for patient safety. N Engl J Med 2003;348:651-6.

24 Berwick DM. Errors today and errors tomorrow. N Engl J Med 2003;348:2570-2. 
25 Ketring SP, White JP. Developing a systemwide approach to patient safety: the first year. Jt Comm J Qual Improve 2002;28:287-95.

26 Moore KA, Coker K, DuBuisson AB, et al. Implementing potentially better practices for improving family centered care in neonatal intensive

care units: successes and challenges. Pediatrics

2003;111:450-60.
27 Joint Commission on Accreditation of Health Care Organizations. Speak up: help prevent errors in your care (hospital brochure). Available at: http:// www.jcaho.org/general+public/patient+safety/speak+up/ hospital+brochure-eng.htm

28 McGuckin M, Waterman R, Storr IJ, et al. Evaluation of a patient-empowering hand hygiene programme in the UK. J Hosp Infect 2001;49:298-9. 